EOMmun Communication et organisation

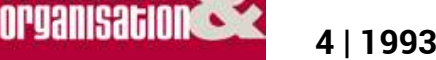

Les relations publiques face à la théorie

\title{
Relations publiques, relations en public
}

Nathalie Heinich

\section{CpenEdition}

Journals

Édition électronique

URL : http://journals.openedition.org/communicationorganisation/1663

DOI : 10.4000/communicationorganisation. 1663

ISSN : $1775-3546$

Éditeur

Presses universitaires de Bordeaux

Édition imprimée

Date de publication : 1 novembre 1993

ISSN : 1168-5549

Référence électronique

Nathalie Heinich, "Relations publiques, relations en public », Communication et organisation [En ligne], 4 | 1993, mis en ligne le 26 mars 2012, consulté le 02 mai 2019. URL : http://journals.openedition.org/ communicationorganisation/1663; DOI : 10.4000/communicationorganisation.1663

Ce document a été généré automatiquement le 2 mai 2019

(c) Presses universitaires de Bordeaux 


\title{
Relations publiques, relations en public
}

\author{
Nathalie Heinich
}

1 Lorsqu'on parle de "relations publiques », on entend implicitement deux choses : d'une part, une forme de liens avec autrui qui concerne non pas des personnes mais des collectifs - une entreprise, une administration ; et d'autre part, un espace de relations qui ne ressortit pas au domaine privé, intime, domestique, mais au domaine public, exposé aux regard, ouvert aux inconnus.

2 Cette double opposition - le public et le privé, le collectif et l'individu - est bien sûr fondamentale, et pertinente pour l'orientation pratique des perceptions et des actions. Mais plutôt que de la traiter comme un clivage entre deux domaines distincts, exclusifs l'un de l'autre, essayons d'y voir un axe de déplacement entre deux pôles opposés. Alors il n'y aura plus séparation entre d'un côté le public et le collectif, de l'autre le privé et l'individuel, les relations publiques et de l'autre les liens entre les personnes ; mais il y aura un même espace, un «champ» relationnel admettant une infinité de positions intermédiaires : des relations plus ou moins publiques ou privées, entre des entités plus ou moins nombreuses - depuis l'unicité de la personne jusqu'à la pluralité de l'entreprise multinationale.

3 Cette perspective n'a rien de révolutionnaire, mais elle aide à penser. Plutôt que dans une logique d'action - qui dichotomise, qui tranche, qui met des frontières, qui opère des discontinuités - installons-nous donc dans une logique de réflexion - qui relie, qui polarise, qui fait des va-et-vient, qui reconstitue des continuités. Cessons pour un moment de circonscrire des camps, et recréons des axes: l'axe du degré d'ouverture (public/privé), et l'axe de la taille des actants (individuel/collectif). Et cessons du même coup de nous préoccuper des «relations publiques» dans l'entreprise, pour nous intéresser à ce qu'est la relation en public : que la forme de cette entrée en relation soit un panneau publicitaire, un exposé dans un séminaire ou une conversation de bistrot; que celui qui entre en relation soit une multinationale, un orateur ou un simple passant; et que celui avec lequel il entre en relation soit un ensemble vaste et indéfini de consommateurs potentiels, un auditoire ou un simple interlocuteur. 


\section{Qu'est-ce alors qu'une « relation publique »?}

Se mettre ou se trouver en relation publique, c'est avant tout s'exposer à autrui. Autrui, d'abord: au maximum, un public multiple et inconnu de celui qui s'expose (c'est par exemple de la campagne publicitaire, mais aussi de l'allocution télévisée) : au minimum, une personne que l'on connaît (c'est par exemple le décideur qu'un commercial doit convaincre, ou la personne aimée que l'on cherche à séduire).

S'exposer, ensuite : ce dernier cas nous suggère que la relation publique ne se réduit pas à être passivement exposé eau regard d'autrui, comme l'est un passant du seul fait qu'il marche dans la rue. Il peut y entrer de la passivité, certes : c'est le cas des «miss » qui s'exposent aux regards du jury dans les concours de beauté. Mais cette passivité n'est qu'apparente, puisqu'elles ne se contentent pas d'être posées la: elles s'exposent, en prenant soin de se présenter sous leur meilleur jour. Voilà qui nous met sur la piste d'une composante essentielle de notre objet : être ou entrer en relation publique, c'est s'exposer au public mais de façon à donner une image de soi aussi favorable que possible - c'est-àdire susceptible de donner envie à autrui de nouer des liens, d'entrer en relations (qu'il s'agisse d'entamer une conversation ou d'acheter le produit d'une marqué). Car dans « relation » il y a une dimension fondamentale de réciprocité - tout aussi important que le « autrui » sous-entendu dans « public ».

6 Une relation publique, donc, c'est cette forme particulière de l'exposition de soi à autrui qui consiste à se présenter d'une certaine façon, à offrir une image qui soit le produit d'un travail de mise en forme, d'une construction de soi dirigée vers l'optimisation de ses ressources à fins de favoriser l'établissement d'une réciprocité, la constitution d'un lien avec l'autre: un regard au minimum, ou encore une parole, un geste, un acte d'appropriation ou de consommation.

7 Cette forme particulière de la présentation de soi, c'est ce qu'on appelle une représentation.

Par-delà la diversité des domaines couverts par ce terme, le sens principal en est à la fois ce qui rend présent (exhibition, notation, description, spectacle) et ce qui supplée à l'absence (délégation, mandat, interprétation). La représentation est donc une opération dialectique, un jeu entre absence et présence, par où un objet absent est rendu «pour ainsi dire » présent - dans ce " pour ainsi dire » se logeant toute la gamme des crédits ou discrédits, la qualité du travail de représentation. Elle comporte donc trois actants: le premier est l'objet absent (représenté : corps, son, texte, grandeur, événement, personne, État, peuple, institution); le deuxième est l'acteur qui produit la mise en présence de l'absent (représentant); le troisième est le destinataire à qui elle est présentée (spectateur, lecteur, sujet, public, instance de décision, contractant). C'est cette interaction entre ces trois actants qui en fait un acte de communication, en tant qu'un objet y transite entre deux sujets, au prix d'un travail spécifique.

La relation en public, c'est donc le fait de s'exposer à autrui en représentation. Si cela vaut d'être précisé, c'est que la représentation n'est pas la seule modalité d'existence : en situation ordinaire on n'est pas la seule modalité d'existence : en situation ordinaire on n'est pas en représentation. On peut être présent, tout simplement, comme lorsqu'on est en tête-à-tête avec soi-même (sauf si l'on se déguise ou si l'on se fait des grimaces devant le miroir), ou comme lorsqu'on est en présence d'autrui sans spécialement songer à 
l'image qu'on est en train de donner de soi, par exemple en marchant dans la rue (sauf si l'on est une star de cinéma qui sait qu'on la reconnait). Ni en représentation, ni simplement présent, on peut être également présenté : «je vous présente monsieur untel »; la présentation ne ressortit pas à la simple présence (sauf si l'on commet l'impolitesse de ne pas répondre à la présentation, en ne saluant pas la personne à qui l'on est présenté); elle ne ressortit pas non plus au travail de représentation (sauf si la présentation se fait sur un plateau de télévision).

Présentation, représentation, présence : ces trois modalités de «l'être-au-monde (pour pasticher les philosophes) peuvent se définir aussi comme trois moments de l'identité, trois façons de se définir. La présentation, c'est ce que j'appellerais, dans le cadre d'une théorie de l'identité, le moment de la désignation, lorsque ce qu'on est nous est renvoyé par autrui qui nous désigne, par exemple, comme l'entreprise X, comme une marqué sérieuse, comme un universitaire ou comme monsieur untel. Cela ne va pas toujours de soi : d'abord parce qu'il existe une multiplicité de désignations possibles (papiers d'identité, bulletin de recensement, instituts de sondage, consommateurs, collègues de travail, amis, conjoint); ensuite parce qu'elles peuvent être jugées inadéquates au sentiment que nous avons de notre propre identité. J'ai par exemple interrogé longuement des écrivains sur ce qu'ils répondent lorsqu'on leur demande ce qu'ils font dans la vie ; et j'ai mesuré l'importance pour eux du moment où, en les désignant comme "écrivain", une instance extérieure (un éditeur, un critique, l'inspecteur des impôts, l'agent de sécurité sociale ou leur voisin de palier) vient conforter leur sentiment intérieur d'en être un; ou à l'inverse lorsque l'absence de cette désignation contredit, souvent douloureusement, ce sentiment; ou encore lorsqu'elle leur paraît déplacée, excessive, culpabilisante, en les gratifiant d'un statut qu'ils n'ont pas le sentiment de mériter.

11 Car à la désignation par autrui s'ajoute cet autre moment de l'identité qu'est l'autoperception - la façon dont un individu se perçoit lui-même, lorsqu'il est amené à se poser la question de ce qu'il est. Ce n'est pas pour autant une simple relation de soi à soi : car la médiation du regard d'autrui est nécessaire pour que se pose la question de l'identité qui sinon se dissout dans le ça-va-de-soi, le flux sans histoires de l'existence quotidienne. C'est, pour le dire vite, la crise identitaire qui fonde ce moment de l'auto-perception : il n'apparait que lorsqu'il y a problème, par exemple lorsqu'il y a décalage avec la désignation - tels les cas d'écrivains vivant comme une injustice leur désignation ou leur non-désignation comme écrivains; tels encore, plus radicalement, les transexuels qui déploient des efforts gigantesques, y compris sur le plan juridique, pour faire coïncider ces deux moments de l'identité sexuelle que sont leur désignation comme hommes et leur autoperception comme femmes (ou le contraire).

On devine dans ce cas combien ces efforts vont se porter sur ce troisième moment de l'identité qu'est la représentation, autrement dit l'image de soi que l'on construit pour soi-même ou pour autrui : ce travail de représentation étant d'autant plus visible lorsque l'écart est grand entre auto-perception et désignation, comme chez les transexuels qui se transforment en travestis pour tenter de faire coïncider ce qu'ils éprouvent, ce qu'ils paraissent et ce qui leur est renvoyé concernant leur identité sexuelle. De même, en disant « je suis écrivain » quelqu'un qui écrit donnera une certaine représentation de luimême, plus ou moins en accord avec la façon dont il se perçoit et dont il est perçu. Et peut-être le dira-t-il d'autant plus qu'il a du mal à faire coïncider ces deux dimensions de son identité professionnelle. 
13 Auto-perception, représentation, désignation : ces trois moments occupent une position spécifique sur l'axe intériorité/extériorité. L'auto-perception est le moment le plus intérieur puisqu'il s'agit d'une relation de soi à soi médiatisée par le langage et l'intériorisation du regard d'autrui; la représentation est un moment intermédiaire, puisqu'il s'agit de l'image offerte à autrui par le sujet; la désignation est le moment de plus grande extériorité, étant le retour de sa propre image donné au sujet par autrui. On voit ainsi le caractère central de la représentation - qui est, rappelons-le, le moment spécifique de la relation en public: elle est ce qui, en évoluant sur l'axe du degré d'intériorité, va pouvoir construire de la cohérence entre l'intériorité de l'autoperception et l'extériorité de la désignation. Parfois, le décalage entre ces moments est faible, de sorte que le travail de représentation sera aussi peu visible pour autrui que peu coûteux pour le sujet; parfois au contraire ce décalage est important, générateur d'une crise d'identité, latente ou patente, qu'un effort constant de représentation va avoir à charge de réduire ou, au minimum, de gérer.

La représentation, du même coup, peut être définie comme un travail de mise en cohérence identitaire. Et la « relation publique » sera probablement d'autant plus réussie que cette cohérence sera mieux assurée : qu'il s'agisse de l'image d'une entreprise et de la confiance qu'on lui accorde, de l'efficacité d'un exposé et de l'intérêt qu'il suscite, ou de la séduction d'une personne et de la sympathie ou du désir qu'elle inspire.

Il y a avantage à parler de mise en cohérence, et à faire intervenir trois moments plutôt que les deux ordinairement perçus selon la logique naïvement dualiste qui tend à opposer la représentation à l'authenticité comme le faux au vrai, le mensonge à la vérité, le rôle à la sincérité, l'artificiel au nature, le social au personnel, le superficiel au profond, l'image à la réalité - etc. Car sortir de ce manichéisme permet de se dégager des jugements de valeur plus ou moins implicites qui orientent la perception de la représentation, comme de tout ce qui touche aux relations publiques.

On s'aperçoit alors que la représentation ne relève pas de ce que Goffman, dans sa "cadre-analyse ", nomme une "fabrication " (c'est-à-dire un dispositif monté à l'insu d'une dupe, comme le sont les pièges, les niches, les opérations d'espionnage, etc.) mais de ce qu'il nomme un "mode ", c'est-à-dire un cadre de l'expérience qui est du rapport ordinaire au monde, s'offrant sous la forme d'un spectacle autorisant le regard frontal: telle une cérémonie, un spectacle théâtral, une rencontre sportive etc.

Dans cette perspective la représentation, dont relèvent les relations en public comme les relations publiques, n'est frappée d'aucune immoralité : elle n'implique pas dissimulation, mensonge ou artifice, mais construction de soi et travail sur son identité - travail et construction qui n'excluent pas à l'occasion le mensonge, l'artifice et la dissimulation, mais à titre de cas extrêmes. Ainsi la publicité peut être perçue non comme une tentative de conditionnement ou d'influence occulte, mais comme un jeu ou une performance, appelant un jugement de valeur selon des critères de qualité esthétique et non pas de moralité ; de même pour le fait de s'exposer en public, comme le fait un acteur ou un orateur; ou encore pour cette forme très ordinaire et très individuelle de représentation qu'est le vêtement, où se jouent subtilement, et parfois à peine consciemment, les microstratégies par lesquelles chacun tente de faire coïncider, par cet instrument essentiel de la représentation de soi, la perception qu'il a de lui-même et la désignation par autrui à laquelle il s'expose. 
C'est la une forme aussi primaire que familière de « relation publique », qui peut aider à faire comprendre, en le libérant de tout jugement de valeur, le travail de représentation ainsi engagé - qu'il s'agisse d'êtres de petite taille comme les personnes, ou de grande taille comme les entreprises; et de situations quasi privées comme les interactions individuelles, ou très publiques comme les campagnes publicitaires ou les opérations de communication à grande échelle. Du stylisme au conseil en communication, il n'y à là que des formes de professionnalisation du travail que nous accomplissons tous, quotidiennement, en nous exposant au regard d'autrui.

\section{RÉSUMÉS}

Cet article propose une réflexion sur la notion de "relations publiques », qui tente de l'élargir au cadre plus général des «relations en public ». Il s'agit alors d'analyser celles-ci comme une forme particulière d'exposition à autrui, et d'examiner les différentes situations où celle-ci peut se produire selon un doublé paramètre : l'axe public/privé, d'une part, et l'axe individuel/collectif d'autre part. La question des relations en public peut ainsi prendre place dans une théorie de la représentation, qui permet d'analyser celle-ci, à l'intérieur des actes de communication, comme un moment spécifique entre présence et présentation, et, à l'intérieur des moments de l'identité, comme un moment spécifique entre auto-perception et désignation. Cette perspective permet de détacher la notion de représentation d'une conception dualiste et manichéenne, et de l'arracher à la condamnation implicite de la fausseté des apparences pour l'étudier comme une forme du travail identitaire : autrement dit, de montrer qu'elle ne relève pas d'une «fabrication » mais d'un « mode », pour reprendre les termes de la cadre-analyse goffmanienne.

This article offers reflections on the notion of "public relations » and tries to widen it to the more general category of «relations in public». It is therefore about analysing these as a particular way of revealing oneself to others, and about examining the different situations where this might take place, according to a dual parameter: the public/ private axis on the one hand, and the individual/collective axis on the other. The question of relations in public can be examined within a theory of representation. which means that they can be analysed within acts of communication, as specific moments between self perception and designation. This perspective allows us to detach the notion of representation from a dualist, manichean conception and to separate it from the implicit falseness of appearances in order to study it as a kink of identity-giving work. In other words, this would show that it is not a question of « fabrication » but rather of « mode » to use Goffman's framework analysis terms.

\section{AUTEUR}

\section{NATHALIE HEINICH}

Nathalie Heinich est sociologue, chargée de recherche au CNRS, membre du Groupe de sociologie politique et morale de l'Ecole des Hautes Etudes en Sciences Sociales à Paris. Titulaire en 1981 d'un Doctorat de L'EHESS consacré à l'histoire de la notion d'artiste, elle s'est spécialisée dans la sociologie des professions artistiques et des pratiques culturelles, 
et a réalisé plusieurs enquêtes sur l'identité d'artiste et la perception esthétique, qui ont donné lieu a de nombreuses publications dans différentes revues. Elle a publié deux ouvrages aux Editions de Minuit : La gloire de Van Gogh ; Essai d'anthropologie de l'admiration (1991) et Du Peintre à l'artiste. Artisans et académiciens à l'âge classique (1993). 